\title{
INTEGRABILITY PROPERTIES OF A GENERALIZED LAMÉ EQUATION: APPLICATIONS TO THE HÉNON-HEILES SYSTEM
}

\author{
Rodica D. Costin \\ Dedicated to my professor, Martin D. Kruskal.
}

\begin{abstract}
We investigate the integrability properties of a second-order linear ODE that is a reduction of the Hénon-Heiles systems and a generalization of the Lamé equation. The equation has four regular singular points in the extended complex plane. The investigation of the poly-Painlevé property (the Kruskal test) is done by calculating explicitly the monodromy group of the equation. The result is that there is only one single-valued and necessarily non-analytic first integral, which is explicitly found using the branching properties of the solutions.

Then, we prove non-integrability of the Hénon-Heiles systems in a comprehensive setting. The method used is the Kruskal test and is general.
\end{abstract}

\section{Introduction}

Recently, Martin Kruskal proposed and applied a new test for integrability. The test, presented in $[1,4,6]$, is a generalization of the Painlevé test, in the sense that movable branch points for the solutions are allowed, as long as the branching of the solutions is not "too rich".

More precisely, consider a first-order ODE in the complex plane

$$
\frac{d x}{d t}=f(x, t)
$$

where $f$ is analytic on a domain $D_{x} \times D_{t} \subset \mathbf{C}^{2}$. The solutions $\mathbf{x}(t)$ may be multivalued, and yet there might exist an analytic first integral in $D_{x} \times D_{t}$ : a non-constant function $F(x, t)$ such that $F(x(t), t)$ is constant for all the branches $x$ of $\mathbf{x}$ in $D_{t}$.

Let us fix some $t \in D_{t}$ and consider the set

$$
S_{t}=\{x(t): x \in \mathbf{x}\} \text {. }
$$

If the set $S_{t}$ is dense in some open set $\tilde{D} \subset D_{x}$ (in which case we say that the solution has bad branching), then any continuous function $F$ which is constant on the trajectories must be constant on $\tilde{D}$, and the equation has no first integrals on $\tilde{D} \times D_{t}$.

This idea is illustrated in [4,6] on Abel's equation $d x / d t=x^{3}+t$, which is shown not to be integrable. In order to study the multi-valuedness of the solutions, the authors study the solution behavior near the singular point $(t=\infty)$ where the branch points may accumulate, giving rise to bad multi-valuedness. The study is performed using

Received November 14, 1995, revised June 5, 1996.

1991 Mathematics Subject Classification: 34A20.

Key words and phrases: non-integrability, Hénon-Heiles system, holomorphic first integral, branching. 
a generalization of the Painleve $\alpha$-method. A small parameter is introduced in the equation, and the series solutions are calculated using a perturbation approach. The terms of the series involve repeated integration on a Riemann surface; by studying the values of these integrals on different paths around the branch points, it is concluded that the solutions are badly branched. Thus, the equation has no first integrals.

The objective of the present research is the investigation of the way this integrability test can be carried out for equations of order larger than 1 .

Section 2 is devoted to the study of the family of second-order linear ODEs

$$
\left(x^{3}-1\right) \frac{d^{2} u}{d x^{2}}+\frac{3}{2} x^{2} \frac{d u}{d x}+\mu x u=0
$$

where $\mu$ is a real parameter. This is a generalization of the Lamé equation, which, in its algebraic form, is [2]

$$
\left(x^{3}-1\right) \frac{d^{2} u}{d x^{2}}+\frac{3}{2} x^{2} \frac{d u}{d x}+\left(h+\frac{n(n+1)}{4} x\right) u=0 .
$$

This equation is known to have a uniform solution if $n$ is a positive integer ( $h$ is a constant, taken to be 0 in (1)).

Equation (1) is also a reduction of the Hénon-Heiles system, which is studied in Section 3.

Since the equation is linear, all the branch points of the solutions are fixed, so a perturbation method does not seem to be productive. Instead, the multi-valuedness of the solutions is described by the monodromy group, and the analysis suggests that the essential obstruction to the existence of integrals is encoded in this group.

In the case of linear equations, while winding around the branch point singularities of the equation, the solutions transform into each other. If the orbit of some solution covers an open set densely in the space of solutions, then this prevents a single-valued continuous (non-locally-constant) function from being constant along the trajectories. In the contrary case, the monodromy group generates level surfaces of functions, which turn out to be integrals of the motion.

In the case of the equation (1), the monodromy group $\mathcal{G}$ is found almost explicitly, and we show that the equation has no global first integrals. (There are, of course, local integrals since the equation is linear; here "global" means that the first integral should be defined on a domain whose projection on the $t$-coordinate contains closed curves surrounding all the singular points.)

More generally, the information about the branching of the solutions in the complex plane is relevant for investigating the existence of real-analytic first integrals, and we use this information to actually calculate the integrals.

The method we use is the study of the action of $\mathcal{G}$ on complex vectors. In abstract terms, we do this by calculating the Lie algebra of (the closure of) the group and by looking at its action on $\mathcal{G}$-invariant functions. The method illustrated on equation (1) is based on the following features of the example treated:

(i) we are able to find the monodromy group (because all the singular points are regular and because the equation has a discrete symmetry), and

(ii) the monodromy group $\mathcal{G}$ acts (on vectors in $\mathbf{C}^{2}$ ) "almost" densely: for generic vectors $c$, the set $\{c G: G \in \mathcal{G}\}$ is dense on a manifold of real dimension 3 in $\mathbf{C}^{2}$, which implies that there are no holomorphic integrals, but that there is a real-analytic one. 
Section 3 is devoted to the study of the Hénon-Heiles system, which is defined by the Hamiltonian

$$
H=\frac{1}{2}\left(\dot{q}_{1}^{2}+\dot{q}_{2}^{2}+a q_{1}^{2}+b q_{2}^{2}\right)+d q_{1}^{2} q_{2}-\frac{1}{3} e q_{2}^{2} .
$$

Different aspects and results on the non-integrability of this system can be found in many papers (see, for example, $[3,5-7]$ ).

The investigation of the Painlevé property shows [5] the existence of solutions with behavior

$$
q_{1}(t) \sim A\left(t-t_{0}\right)^{r_{1}} \quad \text { for } \quad t \longrightarrow t_{0} \quad \text { where } \quad r_{1}=\frac{1}{2} \pm \frac{1}{2} \sqrt{1-48 d / e}
$$

( $A$ is arbitrary), as well as resonances

$$
\begin{aligned}
& q_{1}(t) \sim A_{0}\left(t-t_{0}\right)^{-2}+\cdots+C\left(t-t_{0}\right)^{r_{2}} \text { for } t \rightarrow t_{0} \\
& \text { where } r_{2}=\frac{1}{2} \pm \frac{1}{2} \sqrt{1-24(1+e / d)}
\end{aligned}
$$

( $A_{0}$ has a definite value, $C$ is arbitrary ).

Therefore, the system has the Painleve property only if the numbers $r_{1}, r_{2}$ are integers, which is the case for values of $d / e$ equal to $-1,-1 / 2,-1 / 6$. Integrability also has been proved in the case $d / e=-1 / 16, b=16 a[5]$, for which the numbers $r_{1}$, $r_{2}$ are rational.

Ziglin [7] applies his result on the existence of meromorphic integrals in Hamiltonian systems to the Hénon-Heiles system in the case when $a=b=1, d=-1$; he shows that the system has no meromorphic first integral on the surface of constant energy, for sufficiently low values of the energy.

Starting from Ziglin's theorem, Ito [3] proves a criterion for analytic non-integrability of Hamiltonian systems and applies it to the Hénon-Heiles case, for $a=b \neq 0$ (the inhomogeneity of the potential being important in his treatment). He concludes that the system has a second analytic first integral in $\mathbf{C}^{4}$ only if $d / e=0,-1 / 6,-1 / 2$, and -1 .

In the case $a=b=0$, we prove that if the numbers $r_{1}, r_{2}$ are irrational, then there are no first integrals (independent of the Hamiltonian) under fairly mild conditions on the regularity of the first integrals under consideration. The method used is the Kruskal test, and the approach does not use the Hamiltonian structure; it therefore is suitable for the study of more general systems of ODEs. The approach is rigorous, and it allows for first integrals more singular than the meromorphic functions.

The actual calculation seems to rely on characteristics of the system similar to those used in the proof of Ziglin.

\section{The Kruskal integrability test on a second-order ODE}

Since equation (1) is linear, we can immediately write down two local constants of the motion, as follows.

The points $x=1, x=\omega, x=\omega^{2}\left(\omega^{3}=1\right)$, and $x=\infty$ are regular singular points for the equation. In a neighborhood of $x=1$, there are two independent solutions

$$
\phi(x), \gamma(x)=\sqrt{x-1} \psi(x)
$$


with $\phi, \psi$ analytic. To fix them, we choose $\phi(1)=1, \psi(1)=1$, and the usual branch of the radical.

All the solutions in the complex plane can be written as $u=c_{1} \phi+c_{2} \psi$ for some constants $c_{1}, c_{2}$. Two independent local integrals for the equation can be obtained immediately:

$$
\begin{aligned}
& c_{1}=\frac{u \gamma^{\prime}(x)-u^{\prime} \gamma(x)}{\phi(x) \gamma^{\prime}(x)-\phi^{\prime}(x) \gamma(x)}, \\
& c_{2}=\frac{u^{\prime} \phi(x)-u \phi^{\prime}(x)}{\phi(x) \gamma^{\prime}(x)-\phi^{\prime}(x) \gamma(x)} .
\end{aligned}
$$

The fundamental solutions $\phi, \gamma$ are multi-valued in the complex plane, having branch points at the three singular points of the equation (and so are the local integrals (7)).

In what follows, we will use the expression "the domain $D \subset \mathbf{C}$ permits winding around the points $x_{1}, x_{2}, x_{3}$ " to mean that there is a point $\hat{x} \in D$ different from $x_{j}$, and there are three closed paths $\gamma_{j}$ in $D$, starting and ending at $\hat{x}$, such that $\gamma_{j}$ surrounds only $x_{j}$. (Note that the points $x_{j}$ need not be included in $D$.)

We address the question of existence of global holomorphic and real-analytic first integrals for the equation.

Proposition. Consider the equation (1). Let $\Omega$ be a domain in $\mathbf{C}^{3}$ whose projection on the $x$-coordinate permits winding around the three singular points $x=1, x=\omega$, $x=\omega^{2}$.

If $\mu$ is such that $\sqrt{\frac{1}{16}-\mu}$ is irrational, then

(a) there are no holomorphic first integrals $F\left(u, u^{\prime}, x\right)$ on $\Omega$,

(b) more generally, any holomorphic first integral (in 6 variables) $F\left(u, u^{\prime}, x, \bar{u}, \overline{u^{\prime}}, \bar{x}\right.$ ) (i.e., real-analytic) defined on $\Omega \times \bar{\Omega}$ is a function of

$$
\left|c_{1}\right|^{2}+\tau_{\mu}\left|c_{2}\right|^{2}
$$

where $c_{1}, c_{2}$ are given by (7), and $\tau_{\mu}$ is a real number.

Proof. Step 1. The monodromy group. Consider a fundamental set of solutions near $x=1$,

$$
V_{0}(x)=\left(\begin{array}{l}
\phi(x) \\
\gamma(x)
\end{array}\right),
$$

as in (6). Let $\omega$ be the cubic root of 1 with $\Im \omega>0$. Then $V_{1}(x)=V_{0}(x / \omega)$ and $V_{2}(x)=$ $V_{0}\left(x / \omega^{2}\right)$ are fundamental sets of solutions near $x=\omega$ and $x=\omega^{2}$, respectively, and $V_{0}, V_{1}, V_{2}$ are analytic in a neighborhood of the origin. For $x$ in that neighborhood, define the transition matrices $M, N, P$ by $V_{1}(x)=M V_{0}(x), V_{2}(x)=N V_{1}(x), V_{0}(x)=$ $P V_{2}(x)$. These relations also hold if $x$ is replaced by $x / \omega$ or by $x / \omega^{2}$. It follows that $M=N=P$ and that $M^{3}=I$.

The point at infinity is also a regular singular point, and a fundamental set of solutions near infinity has the form

$$
V_{\infty}(x)=\left(\begin{array}{l}
\phi_{\infty}(x) \\
\gamma_{\infty}(x)
\end{array}\right)
$$


where

$$
\phi_{\infty}(x)=x^{r-\tilde{\phi}}(x), \quad \gamma_{\infty}(x)=x^{r+} \tilde{\gamma}(x)
$$

with $\tilde{\phi}, \tilde{\gamma}$ analytic functions at infinity; $r_{ \pm}$are the characteristic exponents at infinity:

$$
r_{ \pm}=-\frac{1}{4} \pm \nu \quad \text { where } \nu=\left(\frac{1}{16}-\mu\right)^{1 / 2} .
$$

Define the transition matrix $Q$ by $V_{\infty}=Q^{-1} V_{0}$.

Let

$$
A=\left(\begin{array}{cc}
1 & 0 \\
0 & -1
\end{array}\right)
$$

denote the monodromy matrix around a finite singular point and

$$
B=\left(\begin{array}{cc}
e^{2 \pi i r_{-}} & 0 \\
0 & e^{2 \pi i r_{+}}
\end{array}\right)
$$

denote the monodromy matrix at infinity. Then the change of values of the fundamental solutions $V_{0}$ around closed circuits enclosing the singular points is given by the action of the group $\mathcal{G}$ of matrices generated by the monodromy matrices corresponding to loops around each of the three finite singular points: $A$ - corresponding to a loop around $x=1, T=M A M^{2}$ - corresponding to a loop enclosing $x=1$ and $x=\omega^{2}$, and $S=M^{2} A M$ - corresponding to a loop enclosing $x=1$ and $x=\omega$.

The relations among these generators are $M^{3}=I$ and $(A M)^{3}=Q B Q^{-1}$. It turns out that there is almost enough information in these relations to calculate the transition matrices. Simple algebra yields

$$
M=\left(\begin{array}{cc}
\frac{1}{2}\left(z-\Delta^{2}\right) & 1 \\
-\frac{1}{4}\left(z^{2}+3 \Delta\right) & -\frac{1}{2}\left(z+\Delta^{2}\right)
\end{array}\right)
$$

where

$$
z=\omega \alpha_{+}+\delta \alpha_{-}, \quad \alpha_{ \pm}=e^{2 \pi i r_{ \pm} / 3}, \quad \operatorname{det}(M)=\Delta=\omega \delta e^{2 \pi i / 3}, \quad \omega^{3}=\delta^{3}=1 .
$$

Then the generators $S, T$ of $\mathcal{G}$ have the expressions

$$
S=\Delta^{2}\left(\begin{array}{cc}
-\frac{1}{2}\left(z^{2}+\Delta^{2}\right) & -\left(z+\Delta^{2}\right) \\
\frac{1}{4}\left(z^{2}+3 \Delta\right)\left(z-\Delta^{2}\right) & \frac{1}{2}\left(z^{2}+\Delta\right)
\end{array}\right)
$$

and

$$
T=\Delta^{2}\left(\begin{array}{cc}
-\frac{1}{2}\left(z^{2}+\Delta^{2}\right) & -\left(z-\Delta^{2}\right) \\
\frac{1}{4}\left(z^{2}+3 \Delta\right)\left(z+\Delta^{2}\right) & \frac{1}{2}\left(z^{2}+\Delta\right)
\end{array}\right)
$$

Step 2. Let

$$
u(x)=\left(\begin{array}{ll}
c_{1} & c_{2}
\end{array}\right)\left(\begin{array}{l}
\phi(x) \\
\gamma(x)
\end{array}\right)
$$


be a solution of (1) near $x=1$. After traversing a closed circuit around the singular points, the value of the solution becomes

$$
\left(\begin{array}{ll}
c_{1} & c_{2}
\end{array}\right) G\left(\begin{array}{l}
\phi(x) \\
\gamma(x)
\end{array}\right)
$$

where $G$ is the corresponding matrix in $\mathcal{G}$.

Note that, if $u(x)$ satisfies (9), then

$$
\overline{u(x)}=\left(\begin{array}{ll}
\overline{c_{1}} & \overline{c_{2}}
\end{array}\right)\left(\begin{array}{l}
\overline{\phi(x)} \\
\overline{\gamma(x)}
\end{array}\right) .
$$

After traversing a closed circuit in the $x$-plane, if the new value of the solution $u(x)$ is given by (10), then the new value of $\overline{u(x)}$ is

$$
\left(\begin{array}{ll}
\overline{c_{1}} & \overline{c_{2}}
\end{array}\right) \bar{G}\left(\overline{\frac{\phi(x)}{\gamma(x)}}\right) .
$$

Assume that there is a holomorphic first integral: $F\left(u, u^{\prime}, x, \bar{u}, \overline{u^{\prime}}, \bar{x}\right)$, defined on the domain $\Omega \times \bar{\Omega}$.

Let $\left(u_{0}, u_{0}^{\prime}, x_{0}\right)$ be a point in $\Omega$ where $x_{0}$ is not a singular point. Depending on the position of $x_{0}$ in the complex plane, we can write any solution with initial conditions at $x_{0}$ as $u(x)=\left(c_{1}, c_{2}\right) V_{j}(x)$ where $j=0,1,2$, or $\infty$. Here we assume $j=0$ and that the same arguments hold for all other cases. Let $u(x)$ be the solution with $u\left(x_{0}\right)=u_{0}, u^{\prime}\left(x_{0}\right)=u_{0}^{\prime}$. Then (9) holds, for some constants $c_{1}, c_{2}$, denoted, for short, by $c=\left(c_{1}, c_{2}\right)$. We will consider closed paths around the three (finite) singular points of the equation, starting and ending at $x_{0}$.

If $G \in \mathcal{G}$ is any matrix in the monodromy group, then after going on a corresponding path in $\Omega$, we should have

$$
\begin{aligned}
& F\left(c V_{0}\left(x_{0}\right), c V_{0}^{\prime}\left(x_{0}\right), x_{0}, \bar{c} \overline{V_{0}\left(x_{0}\right)}, \bar{c} \overline{V_{0}\left(x_{0}\right)^{\prime}}, \overline{x_{0}}\right) \\
& \quad=F\left(c G V_{0}\left(x_{0}\right), c G V_{0}^{\prime}\left(x_{0}\right), x_{0}, \bar{c} \bar{G} \overline{V_{0}\left(x_{0}\right)}, \bar{c} \bar{G} \overline{V_{0}\left(x_{0}\right)^{\prime}}, \overline{x_{0}}\right)
\end{aligned}
$$

if the point $\left(c G V_{0}\left(x_{0}\right), c G V_{0}^{\prime}\left(x_{0}\right), x_{0}, \bar{c} \bar{G} \overline{V_{0}\left(x_{0}\right)}, \bar{c} \bar{G} \overline{V_{0}\left(x_{0}\right)^{\prime}}, \overline{x_{0}}\right)$ is in the domain of $F$. After a linear change of coordinates, we may omit the fundamental solutions in (11) as well as the fixed point $x_{0}$, and simply write

$$
F(c G, \bar{c} \bar{G})=F(c, \bar{c})
$$

where $F$ is holomorphic in a domain $U \times \bar{U}$.

Consider the following element of $\mathcal{G}: X=(A M)^{3}=Q B Q^{-1}$. Since $\nu$ is irrational, the set $\left\{12 n r_{+}(\bmod 1): n \in \mathbf{Z}\right\}$ is dense in the interval $[0,1] ;$ hence, $\left\{X^{12 n}: n \in \mathbf{Z}\right\}$ is dense in the set

$$
\left\{Q D_{\alpha} Q^{-1}: D_{\alpha}=\operatorname{diag}\left(e^{i \alpha}, e^{-i \alpha}\right), \alpha \in \mathbf{R}\right\}
$$

It follows that

$$
F(c, \bar{c})=F\left(c Q D_{\alpha} Q^{-1}, \bar{c} \bar{Q} \overline{D_{\alpha} Q^{-1}}\right)
$$

also must be true for all $\alpha$ for which $c Q D_{\alpha} Q^{-1} \in U$, therefore, for $\alpha$ small enough. 
Denote $d=c Q$, and let $\tilde{F}$ be defined by $F(c, \bar{c})=F\left(d Q^{-1}, \overline{d Q^{-1}}\right)=\tilde{F}(d, \bar{d})$. Then $\tilde{F}$ satisfies $\tilde{F}(d, \bar{d})=\tilde{F}\left(d D_{\alpha}, \bar{d} D_{-\alpha}\right)$ for $\alpha$ small, or

$$
\tilde{F}\left(d_{1}, d_{2}, \overline{d_{1}}, \overline{d_{2}}\right)=\tilde{F}\left(e^{i \alpha} d_{1}, e^{-i \alpha} d_{2}, e^{-i \alpha} \overline{d_{1}}, e^{i \alpha} \overline{d_{2}}\right) .
$$

Taking the derivative at $\alpha=0$, we get the equation

$$
d_{1} \frac{\partial \tilde{F}}{\partial d_{1}}-\overline{d_{1}} \frac{\partial \tilde{F}}{\partial \overline{d_{1}}}-d_{2} \frac{\partial \tilde{F}}{\partial d_{2}}+\overline{d_{2}} \frac{\partial \tilde{F}}{\partial \overline{d_{2}}}=0
$$

which has arbitrary functions of $\left|d_{1}\right|,\left|d_{2}\right|, \Re\left(d_{1} d_{2}\right)$ as solutions. Thus, any integral has the form

$$
F(c)=\Phi\left(\left|(c Q)_{1}\right|,\left|(c Q)_{2}\right|, \Re\left((c Q)_{1}(c Q)_{2}\right)\right) .
$$

Pick now another element in $\mathcal{G}$ having spectrum on the unit circle, namely $Y=$ $\left(A M^{2}\right)^{3}$. Let $R$ be the matrix which diagonalizes $Y: Y=R(-B) R^{-1}$. By the above argument, there is a function $\Psi$ such that

$$
F(c)=\Psi\left(\left|(c R)_{1}\right|,\left|(c R)_{2}\right|, \Re\left((c R)_{1}(c R)_{2}\right)\right)=\Phi\left(\left|(c Q)_{1}\right|,\left|(c Q)_{2}\right|, \Re\left((c Q)_{1}(c Q)_{2}\right)\right) \text {. }
$$

Denote $c Q=z, P=Q^{-1} R$. Then

$$
\Phi\left(\left|z_{1}\right|,\left|z_{2}\right|, \Re\left(z_{1} z_{2}\right)\right)=\Psi\left(\left|(z P)_{1}\right|,\left|(z P)_{2}\right|, \Re\left((z P)_{1}(z P)_{2}\right)\right)
$$

for all pairs of complex numbers $z$.

Simple algebra yields for the matrix $P$ :

$$
P=\left(\begin{array}{cc}
1+m & m \\
-m & 1-m
\end{array}\right)
$$

where

$$
m=(-1)^{k+l}\left(2 \sin \left(\frac{\pi}{3}(k-l+2 \nu)\right)\right)^{-1},
$$

so $m$ is a real number. The integers $k$ and $l$ are defined by $\omega=\exp (2 k \pi i / 3), \delta=$ $\exp (2 l \pi i / 3)$.

It is convenient to write (13) in polar coordinates: if $z=\left(z_{1}, z_{2}\right)$ with $z_{k}=$ $r_{k} \exp \left(i \theta_{k}\right)$ and if we denote $\theta=\theta_{1}+\theta_{2}, x=\theta_{1}-\theta_{2}$, then the condition (13) becomes

$$
\tilde{\Phi}\left(r_{1}, r_{2}, \theta\right)=\tilde{\Psi}(A, B, C)
$$

where

$$
\begin{aligned}
A= & (1+m)^{2} r_{1}^{2}+m^{2} r_{2}^{2}-2 m(1+m) r_{1} r_{2} \cos (x), \\
B= & m^{2} r_{1}^{2}+(1-m)^{2} r_{2}^{2}+2 m(1-m) r_{1} r_{2} \cos (x), \\
C= & m(1+m) r_{1}^{2} \cos (\theta+x)-m(1-m) r_{2}^{2} \cos (\theta-x) \\
& \quad+\left(1-2 m^{2}\right) r_{1} r_{2} \cos (\theta) .
\end{aligned}
$$

Since the relation must hold for all $x$, by differentiating with respect to $x$ at $x=0$ and at $x=\pi / 2$, we get $\tilde{\Psi}_{C}=0$, and then that $\tilde{\Psi}$ depends only on the combination $(1-m) A+(1+m) B$. Substituting the variable $c=z Q^{-1}$, we get, after a few calculations, that both $\Phi$ and $\Psi$ must be functions of the expression

$$
\left|c_{1}\right|^{2}+\frac{1-m^{2}}{4 m^{2}}\left|c_{2}\right|^{2}
$$


It now can be directly checked that the above expression is invariant under the generators of the monodromy group. The contention (b) of the proposition thus is proved, and so is (a).

\section{The Hénon-Heiles system}

Consider the Hénon-Heiles system (3) for $a=b=0$ :

$$
H=\frac{1}{2}\left(\dot{q}_{1}^{2}+\dot{q}_{2}^{2}\right)-\lambda q_{1}^{2} q_{2}-\frac{1}{3} q_{2}^{3}
$$

(we assume $e=1$ since the variable $q_{2}$ can be rescaled). The corresponding equations of motion are

$$
\begin{aligned}
& \ddot{q}_{1}=2 \lambda q_{1} q_{2}, \\
& \ddot{q}_{2}=\lambda q_{1}^{2}+q_{2}^{2} .
\end{aligned}
$$

Relying on the intuitive picture that the absence of additional first integrals of motion can be due to the presence of almost periodic motions with "badly" related "periods", we choose to study the system in a neighborhood of particular periodic solutions.

Note that there are three two-parameter families of doubly periodic solutions, namely,

$$
\begin{aligned}
& q_{1}=\alpha q_{2}, \\
& \ddot{q}_{2}=\left(\lambda \alpha^{2}+1\right) q_{2}^{2}
\end{aligned}
$$

where $\alpha\left(\lambda \alpha^{2}+1-2 \lambda\right)=0$. After the linear change of coordinates (non-canonical)

$$
q_{1}=\alpha q+U, \quad q_{2}=q,
$$

the equations of motion are

$$
\begin{aligned}
\ddot{U} & =2(1-\lambda) U q-\lambda \alpha U^{2}, \\
\ddot{q} & =2 \lambda q^{2}+2 \lambda \alpha q U+\lambda U^{2} .
\end{aligned}
$$

First take $\alpha$ to be one of the roots of $\lambda \alpha^{2}+1-2 \lambda=0$.

Assume that (18) has a first integral $F(U, \dot{U}, q, \dot{q})$, functionally independent of $H(U, \dot{U}, q, \dot{q})$. We assume that $F$ is meromorphic on a domain $D_{U} \times D_{q}$ and analytic on $\left(D_{U} \backslash(0,0)\right) \times D_{q}$ where $D_{U} \subset \mathbf{C}^{2}$ is a neighborhood of the origin, and $D_{q} \subset \mathbf{C}^{2}$ is a domain whose projection on the $q$-coordinate permits winding around the three cubic roots of some real number. We show that, under these assumptions, the number $r_{2}$ in (5) must be rational. Note that the space of first integrals $F$ allowed is quite large. It contains, for example, functions having essential singularities along analytic varieties. The essential restriction here is the condition that $F$ be meromorphic when $U=\dot{U}=0$ for values of $q$ of all arguments.

In order to perform the Kruskal test, we introduce a small parameter $\epsilon$ by setting $U=\epsilon u$. The system (18) becomes

$$
\begin{aligned}
& \ddot{u}=2(1-\lambda) u q-\epsilon \lambda \alpha u^{2}, \\
& \ddot{q}=2 \lambda q^{2}+2 \epsilon \lambda \alpha q u+\epsilon^{2} \lambda u^{2} .
\end{aligned}
$$


We study the integrability of the reduced system (i.e., at $\epsilon=0$ )

$$
\begin{aligned}
& \ddot{u}=2(1-\lambda) u q, \\
& \ddot{q}=2 \lambda q^{2} .
\end{aligned}
$$

The second equation can be integrated once, and we get

$$
\begin{aligned}
\ddot{u} & =2(1-\lambda) u q, \\
\dot{q}^{2} & =\frac{4 \lambda}{3} q^{3}-K .
\end{aligned}
$$

Note that the constant of integration $K$ corresponds to the Hamiltonian at $\epsilon=0$. We first choose $K$ : let $K$ be such that the projection of $D_{q}$ on the $q$-coordinate permits winding around the three roots of the polynomial $\frac{4 \lambda}{3} q^{3}-K$.

Next, we show that the integral $F$ produces a first integral for (21). Since $F$ is meromorphic, there is an integer $p$ such that

$$
F(\epsilon u, \epsilon \dot{u}, q, \dot{q})=\epsilon^{p} G(\epsilon, u, \dot{u}, q, \dot{q})=\epsilon^{p} \sum_{n=0}^{\infty} \epsilon^{n} G_{n}(u, \dot{u}, q, \dot{q}) .
$$

For $\epsilon$ small, the functions $G_{n}$ have the same regularity as $F$, and the series converges for $(u, \dot{u}, q, \dot{q})$ in the domain of $F$. Then $G_{0}$ is a first integral for the reduced system (20) (or is constant). If $G_{0}$ depends on $u$ or $\dot{u}$, then it is independent of $\left.H\right|_{\epsilon=0}$, hence $G\left(0, u, \dot{u}, q, \sqrt{(4 \lambda / 3) q^{3}-K}\right)$ is a first integral for (21).

If $G_{0}$ depends only on $(q, \dot{q})$ or is constant, then this reduces to the preceding case in the following way. The function $G_{0}$ will be an integral for the second equation in (20) (or a constant); therefore, $\epsilon^{-p-1}\left(F-G_{0}\right)$ is either a first integral for (21) or a constant. We repeat the procedure, until, for some $n_{0}, G_{n_{0}}$ depends on $u$ or $\dot{u}$. (There is such a number $n_{0}$ because (19) obviously does not admit integrals depending only on $(q, \dot{q})$.) The upshot is that there exists a first integral $G(u, \dot{u}, q, \dot{q})$ for $(21)$, having the same regularity as $F$.

The next step is eliminating the time in (21). A formal calculation gives

$$
\frac{d^{2} u}{d q^{2}}\left(\frac{4 \lambda}{3} q^{3}-K\right)+2 \lambda q^{2} \frac{d u}{d q}+2(\lambda-1) q u=0 .
$$

It is clear that $G\left(u, \frac{d u}{d q} \sqrt{(4 \lambda / 3) \lambda q^{3}-K}, q, \sqrt{(4 \lambda / 3) \lambda q^{3}-K}\right)$ is a first integral for (22). Denote it by $\tilde{G}\left(u, u^{\prime}, q, \sqrt{(4 \lambda / 3) \lambda q^{3}-K}\right)$ (here $u^{\prime}$ stands for the $q$-derivative of $u$ ). By restricting $\tilde{G}$ to a sub-domain $\tilde{D}_{U} \times D_{q}$, we may assume that $\tilde{G}$ is analytic in $\left(u, u^{\prime}, q, \dot{q}\right)$.

Rescaling the variable $q$ by

$$
q=\left(\frac{3 K}{4 \lambda}\right)^{1 / 3} x
$$

the equation (22) becomes (1) with $\mu=3 / 2(1-1 / \lambda)$ and

$$
\tilde{G}\left(u, u^{\prime}, q, \sqrt{\frac{4 \lambda}{3} \lambda q^{3}-K}\right)=\hat{G}\left(u, u^{\prime}, x, \sqrt{x^{3}-1}\right)
$$

where $\hat{G}$ is analytic in four variables on $\tilde{D}_{U} \times D_{x}$ (where, clearly, $D_{x}$ is a dilation of $\left.D_{q}: D_{x}=(3 K / 4 / \lambda)^{-1 / 3} D_{q}\right)$. 
In section 2 , it was shown that if $\sqrt{1 / 16-\mu}$ is irrational, then there are no first integrals for (1). In that argument, only paths surrounding the singular points an even number of times are used. Therefore, the argument also applies to first integrals of the type $\hat{G}\left(u, u^{\prime}, x, \sqrt{x^{3}-1}\right)$. Hence, $r_{ \pm}$given by (8) must be rational. Note the connection between the numbers $r_{ \pm}$and the resonances $r_{2}$ from (5): $r_{ \pm}=-r_{2} / 2$. Hence, $r_{2}$ must be rational.

Consider now the value $\alpha=0$ (the change of coordinates (17) is trivial in this case). Assume that there is a second first integral $F$ with the same properties as before. Substituting $U=\epsilon u$, the system (18) becomes

$$
\begin{aligned}
& \ddot{u}=2 \lambda u q, \\
& \ddot{q}=q^{2}+\epsilon^{2} \lambda u^{2} .
\end{aligned}
$$

As before, we study the reduced system which is, after one integration,

$$
\begin{aligned}
\ddot{u} & =2 \lambda u q, \\
\dot{q}^{2} & =\frac{2}{3} q^{3}-K .
\end{aligned}
$$

Eliminating the time and rescaling, we get the equation (1) for $\mu=-3 \lambda$, which has a first integral.

Since, in this case, the connection between the numbers $r_{ \pm}$and the resonances $r_{1}$ given by (4) is $r_{ \pm}=-r_{2} / 2$, it follows that $r_{1}$ must be rational.

\section{Conclusions}

We have proved that if one of the resonances $r_{1}, r_{2}$ is irrational, then there are no additional first integrals $F\left(q_{1}, \dot{q}_{1}, q_{2}, \dot{q}_{2}\right)$ such that $F$ is meromorphic on a domain $D_{1} \times D_{2}$, analytic on $\left(D_{1} \times D_{2}\right) \backslash V_{\alpha} \backslash V_{0}$ where $V_{\alpha}$ is the variety $\left\{q_{1}=\alpha q_{2}, \dot{q}_{1}=\alpha \dot{q}_{2}\right\}$ and $V_{0}$ is the variety $\left\{q_{1}=0, \dot{q}_{1}=0\right\}$, and the projection of $D_{2}$ on the $q_{2}$-coordinate permits winding around the three roots of a real number. In the notations (15), the condition that $r_{1}$ or $r_{2}$ be irrational is: $\sqrt{1+48 \lambda} \in \mathbf{R} \backslash \mathbf{Q}$, or $\sqrt{-23+24 / \lambda} \in \mathbf{R} \backslash \mathbf{Q}$.

The analysis above is not exhaustive: there still is more information to be deduced from the monodromy group of the equation (1). This could answer the question whether there also exist rational or non-real values of $\nu$ for which the equation is not integrable in the complex domain. Also, the Kruskal test was performed only to leading order, and to that order there exists a real-analytic first integral (14) (besides the reduced Hamiltonian), which is in fact an integral in the real domain. The multivaluedness of the solutions needs to (and can) be studied to the next orders to possibly rule out the existence of an integral in the real domain.

Acknowledgments. I would like to express my profound admiration and gratitude to my professor, Martin Kruskal, who introduced me to the fascinating world of nonintegrability. I am extremely grateful to Professor Joel Lebowitz for proposing a very interesting line of research and for his permanent care, and to my professor François Treves, for very interesting discussions and his continuous, invaluable encouragement.

\section{References}

1. M. D. Kruskal, A. Ramani, and B. Grammaticos, Singularity analysis and its relations to complete, partial and non-integrability, In: Partially Integrable Nonlinear Evolution Equations and Their Physical Interpretation, (R. Conte ed.), Kluwer Acad. Publ., Dordrecht, (1990). 
2. E. L. Ince, Ordinary Differential Equations, Dover, New York, 1956.

3. H. Ito, A criterion for non-integrability of Hamiltonian systems with nonhomogeneous potentials, J. Appl. Math. Phys. 38 (1987).

4. M. D. Kruskal and P. A. Clarkson, The Painlevé-Kowalevski and poly-Painlevé test for integrability, Stud. App. Math. 86 (1992), 87-165.

5. B. Grammaticos, B. Dorizzi, and R. Padjen, Painlevé property and integrals of motion for the Hénon-Heiles system, Phys. Lett. 89A.

6. A. Ramani, B. Grammaticos, and T. Bountis, The Painlevé property and singularity analysis of integrable and non-integrable systems, Phys. Repts. 180 (1989), 159-245.

7. S. L. Ziglin, Branching of solutions and the non-existence of first integrals in Hamiltonian mechanics I, II, Func. Anal. Appl. 16 (1983), 181-189; 17 (1983), 6-17.

Department of Mathematics, Rutgers University, Hill Center, Busch Campus, Piscataway, NJ 08854

E-mail: rcostin@math.rutgers.edu 